\title{
IMPLEMENTACIÓN DE UNA ESTRATEGIA DE CONTROL DIFUSO PARA AUMENTAR LA PRODUCCIÓN DE CRUDO EN POZO PETROLERO
}

\section{IMPLEMENTING A FUZZY CONTROL STRATEGY TO INCREASE OIL PRODUCTION IN THE WELL}

\author{
PhD. Oscar Eduardo Gualdrón Guerrero, MSc. Juan José Reales Osorio. \\ MSc. Tania Liseth Acevedo Gauta.
}

Universidad de Pamplona, Facultad de Ingenierías y Arquitectura. Ciudadela Universitaria. Pamplona, Norte de Santander, Colombia.

Tel.: (+577) 5685303, Fax: (+577) 5685303, Ext. 144.

E-mail: oscar.gualdron@unipamplona.edu.co, \{jreales27, t1_acevedo\}@hotmail.com

\begin{abstract}
Resumen: En este trabajo se plantea una estrategia de control enfocada en la producción de crudo en un sistema de bombeo electro-sumergible, utilizando un controlador difuso implementado en un PLC. Para la etapa de identificación del proceso se adquiere los datos de un pozo de producción de crudo modelando el sistema y así estableciendo las variables que determinan el proceso. Se realiza el diseño y ajuste del controlador difuso por Matlab, para implementar el controlador difuso utilizado el software Fuzzy Designer y un PLC ControlLogix L61.
\end{abstract}

Palabras clave: Identificación y modelado de sistemas, Control difuso, control PID,

PLC, producción de crudo.

\begin{abstract}
This paper presents a control strategy that improves oil production in an electro-submersible pump, using a fuzzy controller implemented in a PLC. For the identification stage of the process is acquired as the reference data of a production well with her crude modeling the system and thus setting the variables that determine the process, design and setting of fuzzy controller is performed by Matlab, and then implement the fuzzy controller used the Fuzzy Designer software from Rockwell Automation ControlLogix L61 and a PLC.
\end{abstract}

Keywords: System identification, fuzzy control, PID control, PLC, oil production.

\section{INTRODUCCIÓN}

Dentro del proceso de extracción del petróleo se encuentra la primera etapa que es el bombeo de crudo del yacimiento a la superficie por medio de técnicas de levantamiento artificial BES (Bombeo Electro Sumergible).

Estos sistemas son importantes debido a que es el punto de partida de la cantidad de producción que pueda suministrar un pozo.
Cada pozo posee un control de afluencia (CAP), este control efectúa eventos o sugerencias por medio del SCADA para el cambio de frecuencias del variador de velocidad del pozo, este proceso es verificado por personal experto que analiza el comportamiento del yacimiento, para que de esta manera realice la toma de decisiones para el control de velocidad, siendo esta estrategia de control no muy adecuada para controlar la afluencia del pozo (CAP), ya que es un control OnOff alrededor de la variable PIP. 


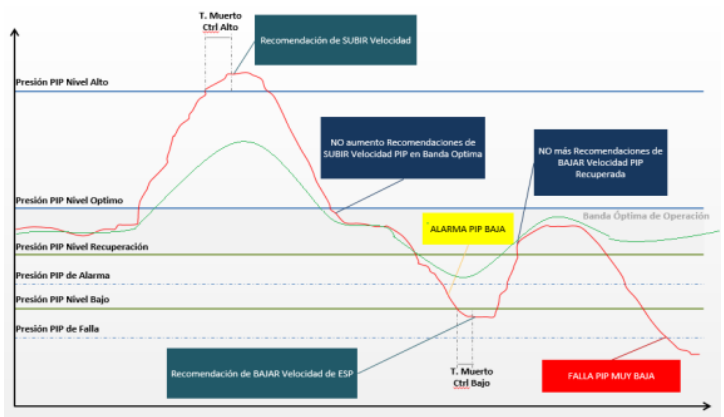

Fig. 1. Comportamiento de la variable PIP, línea roja control On-Off de la PIP, línea verde Control Difuso de la PIP.

Lo que se quiere con este trabajo es por medio del control difuso mejorar las respuestas de control y de esta manera suministrar las recomendaciones más oportunas en pro de la producción de petróleo.

\section{DESCRIPCIÓN DEL PROCESO}

El proceso de extracción de crudo tiene como finalidad garantizar que el fluido se bombee a la superficie con suficiente presión para romper la resistencia de la gravedad y la trocal.

\subsection{Descripción del proceso asociado a la extracción de crudo}

Con los datos de campo del área o pozo y con el monitoreo constante de la presión de entrada a la bomba (PIP), se puede visualizar la curva de afluencia del pozo y la presión de fondo fluyente (PWF).

Con ello se permite determinar y mantener una tasa de flujo constante en el pozo a través del control de la velocidad de la bomba optimizando el funcionamiento del sistema.

La filosofía del control y optimización del sistema de levantamiento por bombeo electro sumergible (BES) es mantener una afluencia constante del pozo, basado en el control directo de la PIP con el ajuste de la velocidad de la bomba; es decir un control On-Off alrededor de la variable de control.

En la Figura 1.se muestra como es la filosofía del CAP (control de afluencia de pozo) la cual controla la variable PIP (presión a la entra de la bomba), este actúa por zonas o franjas de control, donde realiza acciones de control con la velocidad del variador $O n$-Off alrededor de la variable.

\section{METODOLOGÍA}

Para realizar la identificación del sistema se realiza un estudio de las variables del proceso, determinando cuáles son las más influyentes en el mismo, que por ende son las que más información aportan para realizar su identificación.

Una vez realizado este estudio se lleva a cabo la toma de datos. Con el conjunto de datos depurado se puede determinar su modelo de identificación y finalmente desarrollar estrategia de control clásico y difuso para la extracción de crudo.

\subsection{Análisis de las variables para realizar la identificación del sistema}

Las variables más representativas que están implícitas en el proceso son temperatura, presión y flujo, de estas, después de realizar el análisis se logró determinar que cada una aporta las siguientes características:

Temperatura: Tiene consecuencias en la viscosidad del crudo nos indica la fluidez del pozo velocidad de descarga a línea de producción.

Flujo: Variable que es importante para que tener la referencia la producción del pozo y cuanto aporta a la troncal.

Presión: Esta variable reviste importancia, nos suministra datos tales como la presión con la cual esta entrado al manifold y presión de fondo del fluido siendo clave para calcular la sumergencia del pozo que es a su vez la variable a controlar para saber los ajustes de control que va tener nuestro sistema. Debido a esto es porque se elige como el centro de nuestro estudio.

\subsection{Recolección de la Data}

Se determinó que la variable que define el comportamiento del sistema es la presión de fondo (PIP). Para la obtención de la data correspondiente a esta variable se programó un historial en el servidor del SCADA con un tiempo de $40 \mathrm{Seg}$ de muestreo Figura 2. 


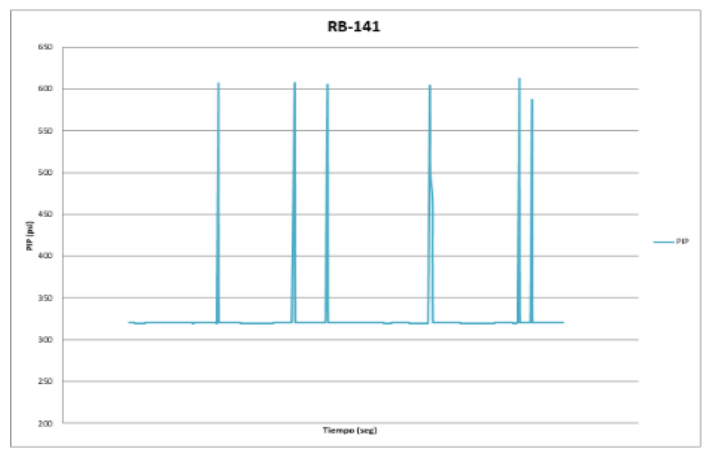

Fig. 2. Gráfica de la data recolecta.

\subsection{Identificación del proceso}

Para identificar el sistema se escogió el método de la curva de reacción de Ziegler Nichols, debido a que el sistema presión presenta características dinámicas que nos permiten aproximarlo a un sistema de primer orden con tiempo muerto y con la consideración de que el tiempo muerto $t_{0}$ del sistema es 0 dado que no poseemos el Euler se cancela y remplazando en ecuación (1)

$$
\frac{\Delta o(s)}{\Delta i(s)}=\frac{k e^{-t_{0} s}}{t s+1}=\frac{3.72}{200 s+1}
$$

Ahora las ecuaciones (2), (3) y (4) de ajustes para el controlador PID

$$
\begin{aligned}
& k_{c}=\frac{1.2}{3.72}\left(\frac{45.5}{157.5}\right)^{-1}=1.2 \\
& t_{i}=2.0 \cdot 42.5=85 \\
& t_{d}=0.5 \cdot 42.5=21.75
\end{aligned}
$$

Lazo de control de la presión de fondo en el proceso de extracción de crudo Figura 3

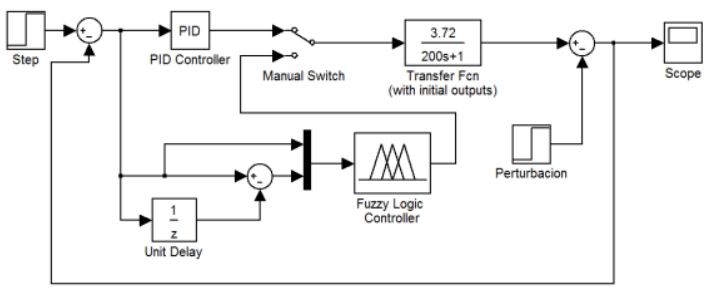

Fig. 3. Esquema de control implementado en Simulink.

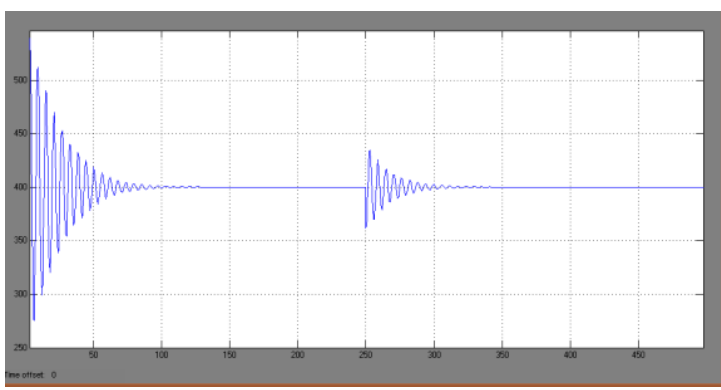

Fig. 4. Respuesta del controlador PID implementado en Simulink.

Observado la figura 4 se evidencia que no se obtuvo una respuesta adecuada del sistema, dado que se presenta una oscilación muy pronunciada al comienzo de la acción de control, esta respuesta es una acción de control no idónea para nuestra aplicación ya que no se puede arrancar de esta manera el motor por que puede ocasionar un daño en el mismo, también se observa que al momento del sistema es perturbado a los $250 \mathrm{mseg}$ se presenta la misma oscilación. Entonces para este proceso se tiene que replantear una estrategia de control diferente.

\subsection{Replanteamiento de la solución}

Se procede a modificar la estrategia de control a utilizar con la siguiente propuesta que es realizar un controlador lógico difuso (CLD), que sería más apropiado para el manejo de la oscilación del sistema, esto es debido a que las oscilaciones y perturbaciones están y a en la base de conocimiento (BDC), del CLD. Dado que conocemos el proceso y las pautas de funcionamiento, la experiencia del experto resultaría práctico realizar un sistema basado en reglas para la toma de decisiones. Tal tipo de procesamiento, está mucho más cercano al razonamiento del experto humano, considerando que es necesario agregar a los programas ese conocimiento y experiencia.

Funciones de pertenencia de la variable error Sobre el universo de variación del variable error Figura 5 se establecieron 5 conjuntos difusos, cada una de las variables lingüísticas en las que se subdivide el universo de variación del error fueron creadas a través de entrevistas con operadores del proceso y un estudio del mismo. 


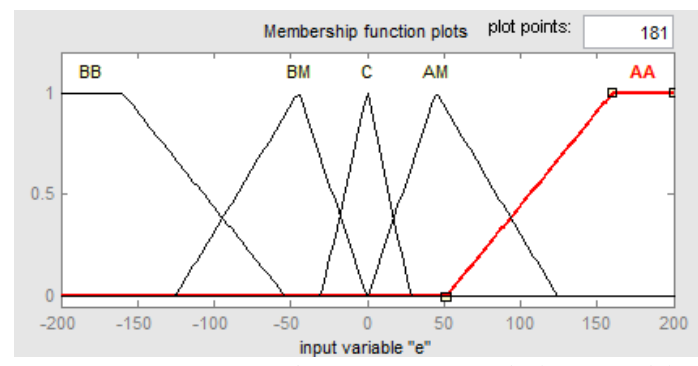

Fig. 5. Funciones de pertenencia de la variable error-Simulink, Matlab.

Funciones de pertenencia de la variable derivada del error se mediante las especificaciones del operador experto.

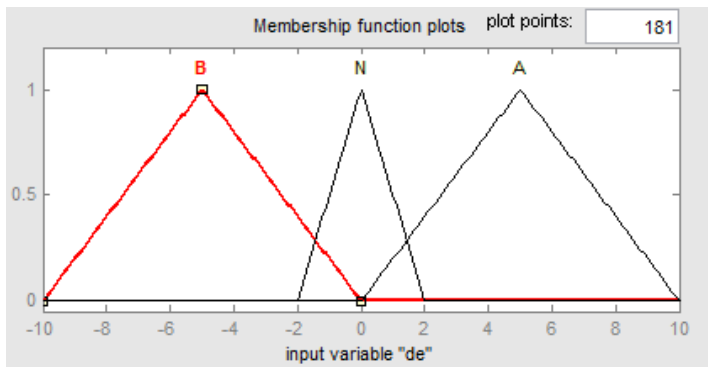

Fig. 6. Funciones de pertenencia de la variable derivada error-Simulink, Matlab.

Funciones de pertenencia de la señal de salida Figura 7 del controlador lógico difuso. La señal de salida del controlador debe variar en un rango de 0 a 400. El actuador lo constituye la acción de control hacia el cambio de frecuencia del variador. Que tiene una relación lineal entre el voltaje de entrada.

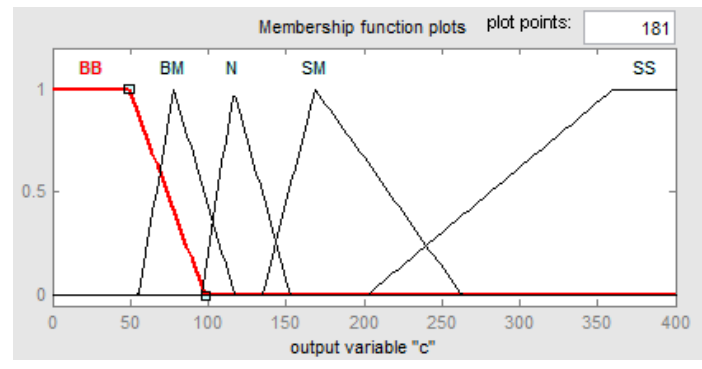

Fig. 7 Funciones de pertenencia de la señal de salida del CLD-Simulink, Matlab.

$\underline{\text { Tabla 1. Reglas del controlador difuso }}$

\begin{tabular}{lccccc}
\hline $\begin{array}{c}\text { Error } \\
\text { Derivada } \\
\text { Error }\end{array}$ & B B & BM & C & AM & AA \\
\hline B & BM & BM & N & N & SS \\
N & BB & BM & N & SM & SS \\
A & BB & N & N & SM & SM \\
\hline
\end{tabular}

Todas estas reglas (Tabla1) se expresan mediante sentencias del tipo "sie is $X 1$ and $Y$ is $X 2$ then $S$ es $A x$ ”. La unión de todas las reglas es lo que formará la base de conocimiento del controlador difuso. Esto se logra matemáticamente mediante el operador conectivo OR que en nuestro caso es el del máximo pero pudiera ser cualquier Snorma. Entonces la experiencia total del experto estará formada por la unión de todas las reglas.

Por el programa de simulación Simulink de Matlab se da la tarea de comparar las respuestas del controlador PID y el CLD. Con el fin de constatar que el CLD tiene una respuesta de control mucho más adecuada para Figura 8.

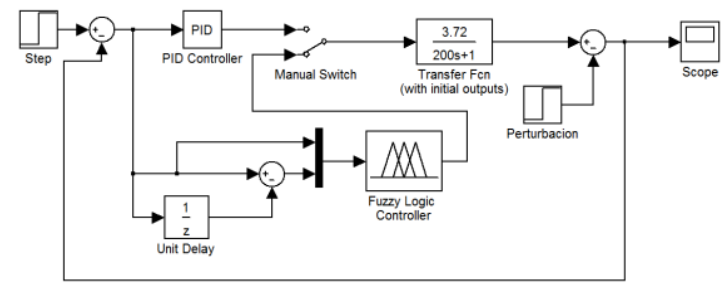

Fig. 8. Esquema de control difuso implementado en Simulink.

Se realizan distintas pruebas con el fin de demostrar que el controlador lógico difuso está trabajando óptimamente y que puede controlar la oscilación que tienen el control PID.

El CLD Figura 9 si responde adecuadamente al set point de 400 psi que se le ingresa dando una curva de estabilización y llegando a 402 psi con una ligera diferencia en la salida con el set point dándole un rango aceptables, además de una perturbación de 50 psi a los $250 \mathrm{mseg}$.

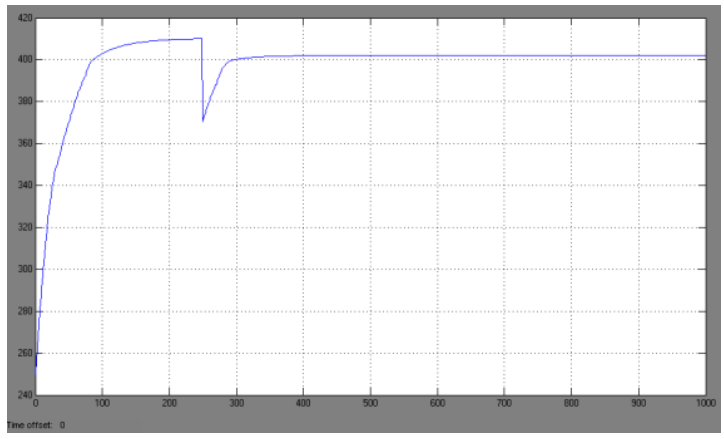

Fig.9 Respuesta del control difuso implementado en Simulink con un step de 400 y una perturbación de 50 a los 250 mseg. 


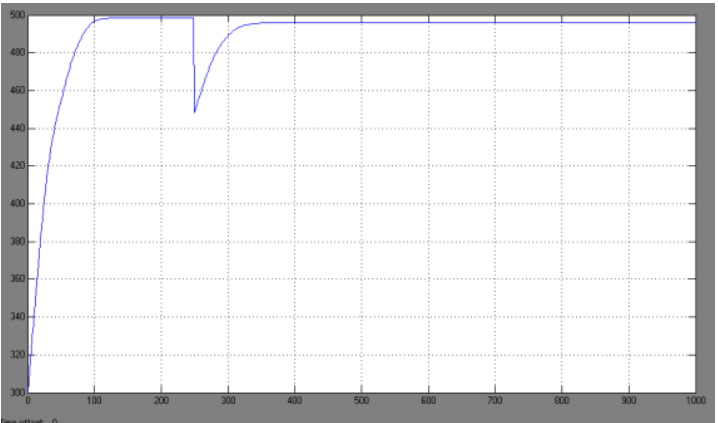

Fig. 10. Respuesta del control difuso implementado en Simulink con un step de 500 y una perturbación de 50 a las $250 \mathrm{mseg}$.

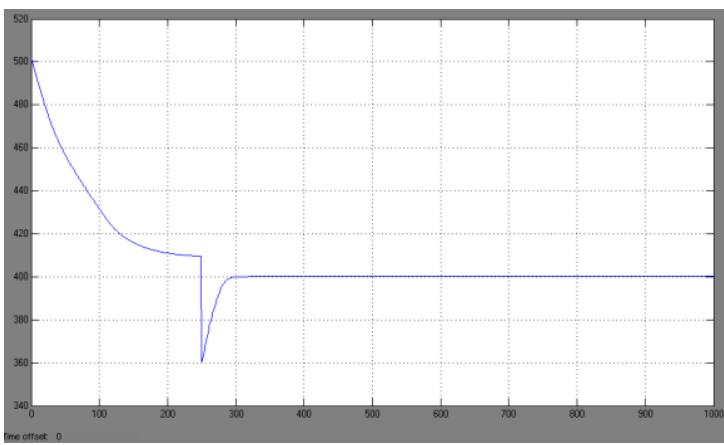

Fig. 11. Respuesta del control difuso implementado en simulink con un step de 400 y una perturbación de 50 a los $250 \mathrm{mseg}$.

Con las respuestas que se representan en las figuras 10 y 11 anteriores se demuestra que el controlador difuso si es idóneo para manear esta planta, incluso con perturbación el sistema responde.

También se cambió a diferentes set point para verificar que este funcionado correctamente, no obstante cabe resaltar que este controlador tiene pequeñas diferencias en la salida con el set point de entrada de 0 a 5 psi.

Se puede deducir que el controlador PID no cumple las expectativas y requerimientos del sistema por lo cual se optó por un control inteligente lógico difuso donde es más práctico para este tipo de procesos donde predomina la experiencia.

Se tomaron los datos PIP vs Tiempo como parámetro para encontrar la planta, ya que el experto toma decisiones en base a esas variables para realizar las acciones de control.

Estos datos obtenidos obedecen a un comportamiento de un día aleatorio de trabajo determinado del pozo, esto quiere decir desde el punto de vista estadístico que los datos tomados fueron una muestra aleatoria de la población, en donde en este caso la población representa el comportamiento del proceso de extracción de crudo del yacimiento.

\section{RESULTADOS}

Para la implementación del controlador difuso en un PLC Allen Bradley y validar el funcionamiento del controlador se consta del software FuzzyDesigner de Rockwell Automation para el diseño de sistemas difusos. Un sistema difuso creado en fuzzy designer puede ser exportado a los proyectos de los controladores de la familia RSLogix 5000 de Allen Bradley este posee un entorno de programación de diagrama en bloques haciendo la programación intuitiva.

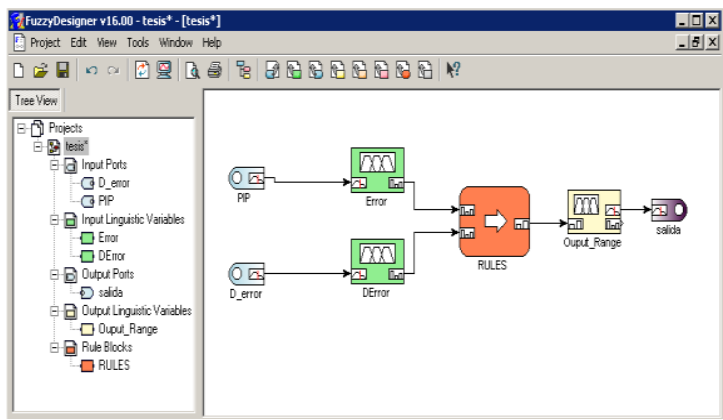

Fig. 12. Diseño del controlador difuso en software FuzzyDesigner v16.

En respuesta que nos muestra Figura. 13, se evidencia que el controlador está funcionando a cabalidad como se esperaba, estabilizando al 400 PSI en un tiempo de 62 (seg), se realizó unos ajustes on-line de los rangos de las membresías y conjuntos difusos buscando la mejor respuesta del controlador.

SHistory Graph - Control_PIP

$-\bar{a} \mid \mathrm{x}$

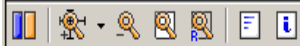

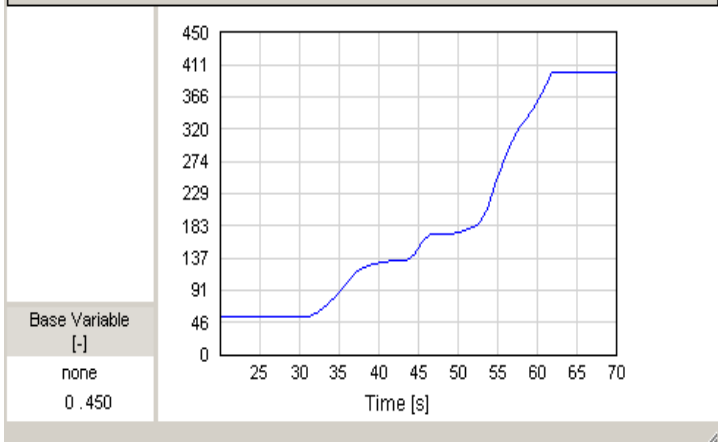

Fig. 14. Respuesta de control difuso implementado en el PLC. 


\section{CONCLUSIONES}

El sistema de control de afluencia de pozo que se diseñó con la estrategia de control PID no cumplió las expectativas esperadas, dado que poseía una oscilación al comienzo de la acción de control no aceptable para el tipo de aplicación a la que se quiere.

A este problema se le pudo dar solución proponiendo un controlador lógico difuso que es más apropiado para el manejo de la oscilación del sistema, esto es debido a que las oscilaciones y perturbaciones están ya en la base de conocimiento (BDC), del CLD.

Dado que conocemos el proceso y las pautas de funcionamiento, la experiencia del experto resulta práctico tener un sistema basado en reglas para la toma de decisiones. Tal tipo de procesamiento, está mucho más cercano al razonamiento del experto humano.

La respuesta de la salida del controlador difuso en el PLC Allen Bradley fue la esperada, llevando la señal de acción de control al Set Point esperado. Sin embardo se precisa ajustar la respuesta por medio del software Fuzzy Designer V16 de Rockwell Automation On-line para llevarla a los rangos deseados.

Esto ratifica la rápida respuesta del sistema ante un cambio significativo de la variable de control. Enviando más pronto las sugerencias y eventos al SCADA para su análisis y posterior validación. Esto hace que se ahorre tiempo en estudio y se aumente la producción de crudo.

\section{REFERENCIAS}

Zdenko Kovacic, Stjepan Bodgan, Fuzzy Controller Design, Theory and Applications

M. I. Alberto Pedro Lorandi Medina, "Controladores PID y Controladores Difusos", Revista de Ingeniería Industrial, Vol. 5, No. 1, 2011.

Tarantino Alvarado, R. "Curso de Identificación de Sistemas", Pamplona, Colombia, Universidad de Pamplona, 2011.

Juan Carlos Gómez, "Fuzzy Control", Grupo de Inteligencia Artificial y Robótica, Secretaría de Ciencia y Tecnología, Universidad Tecnológica Nacional - FRBA, 2008

Efrain E. Barnerii, El pozo ilustrado, FOCIED. Venezuela, 1998

Sebastian Beca Cofre, "Clustering difuso con selección de atributos", Tesis de Maestría, Universidad De Chile, Facultad De Ciencias Físicas y Matemáticas, 2007.

Pardo G. Aldo, Díaz R., Jorge Luis. Fundamentos en Sistemas de Control Automático Universidad de Pamplona. 1ra Edición., pp. 3744, Colombia, 2004.

Creus Sole. Antonio. "Instrumentación Industrial". Marcombo-Boixareu Editores, $7^{\mathrm{ma}}$ Edición, 1996, pp.775.

Fernando Otero, "Fundamentos de Control de Procesos para la Industria Petrolera - peradores - XY”, Optimo Group Inc.,2013

The MathWorks, Inc., 1994 - 2011, Available on the Internet: http://www.mathworks.com/products/fuzzylogic/description $3 . \mathrm{html}$

Publication LOGIX - UM004A - EN - P - March 2007, Rockwell Automation, Inc. RSLogix5000 FuzzyDesigner, U.S.A., 2007. Available on the Internet: http://literature.rockwellautomation.com/idc/gr oups/literature/documents/um/logixum004_-enp.pdf

Siemens AG, 1998. Simatic S7 Fuzzy Control, Germany, 1998. Available on the Internet:http://cache.automation.siemens.com/d n1/TU1Nzg 1NwAA_1135654_HB/Fuzzy 